\title{
Motivação para a prática de atividades físicas e esportivas de crianças: uma revisão sistemática
}

\author{
Motivation for the practice of physical activities and sports of children: a \\ systematic review
}

Gisele Graziele Bento', Elizandra Gonçalves Ferreira', Franciele Cascaes da Silva', Paulo Henrique Mattana', Rudney da Silva

\section{Resumo}

Este estudo teve como objetivo revisar sistematicamente a produção científica sobre motivação para prática de atividades físicas e esportivas de crianças. Para isso, foram utilizadas as bases de dados: MEDLINE via Pubmed, Scielo, Web of Science e SCOPUS (Elsevier) e a estratégia de busca incluiu os descritores propostos no Medical Subject Headings (MeSH) e seus correlatos: "Motivation", "Disincentives", "Disincentive", "Incentives", "Incentive”, "Sports", "Sport”, "Athletics", "Athletic", "Motor Activity", "Child", "Children". Foram incluídos artigos científicos originais de base populacional ou escolar sem restrição de local e idioma; com amostra de crianças; com temática sobre motivação para a prática de atividade física e esportiva de crianças; com resumos e textos completos disponíveis na íntegra pelo meio on-line; publicados nos últimos dez anos. Identificou-se que a motivação para a prática de atividades físicas e esportivas é uma temática abordada em todo o mundo, mas observa-se pouca produção específica a respeito no Brasil, apesar da produção científica da área vir aumentando nos últimos anos. Foram incluídos dezoito estudos, os quais demonstraram que a maior parte das crianças valorizavam amizade, manutenção do físico, saúde e peso e status social como aspecto motivacional para a prática de atividades físicas e esportivas, sendo que as meninas valorizavam mais amizades, diversão e manutenção do físico, saúde e peso enquanto os meninos valorizavam mais o desempenho e habilidades, competição, status social e amizades. Assim, devem-se elaborar estratégias de intervenção que abordem tais aspectos, visando a continuidade de participação dos sujeitos.

\section{Palavras-chave}

Motivação; Atividade motora; Esportes; Criança.

\begin{abstract}
This study aimed to systematically review the scientific production about motivation for the practice of physical activities and sports of children. Therefore, the databases were used: MEDLINE via PubMed, Scielo, Web of Science and Scopus (Elsevier) and the search strategy included the descriptors proposed in Medical Subject Headings (MeSH) its related: "Motivation," "Disincentives" "Disincentive", "Incentives", "Incentive", "Sports", "Sport", "Athletics", "Athletic", "Motor Activity", "Child", "Children". Composed of original scientific articles of population base or school base without restriction of place and language; with sample of children; with theme on motivation for physical activity and sport for children; with abstracts and full texts available online in free access; published in the last ten years. Identified that the motivation for the practice of physical activities and sports is a subject discussed all over the world, but there has been little production specific in Brazil, despite the scientific production of the subject increase in recent years. Eighteen studies were included, which showed that most children valued friendship, physical maintenance, health and weight and social status as a motivational aspect to the practice of physical activities and sports, and girls valued more friendships, fun and maintenance physical, bealth and weight while boys more importance to performance and skills, competition, status and friendships. Thus, should develop intervention strategies that approach these aspects, in order to continue participation of the subjects.
\end{abstract}

\section{Keywords}

Motivation; Motor Activity; Sports; Child

\section{Introdução}

Os comportamentos sedentários estão cada vez mais presentes nas primeiras fases da vida, especialmente

1 Universidade do Estado de Santa Catarina, Centro de Ciências da Saúde e do Esporte, Laboratório de Atividade Motora Adaptada. Florianópolis, SC, Brasil. nas sociedades industrializadas, os quais aliados à inatividade física contribuem de forma substancial para a piora dos estados gerais de saúde ${ }^{1}$. Considerando que os comportamentos adquiridos na infância tendem a permanecer na fase adulta, faz-se necessária a investigação dos motivos que levam à adoção 
de práticas de atividade física nas fases iniciais da vida, reconhecendo as transformações dos diferentes determinantes deste processo ${ }^{2}$.

A inatividade física está associada a determinantes psicológicos, sociais, econômicos e culturais ${ }^{3-7}$. Entre os fatores psicológicos, pode-se destacar a motivação como primordial para a manutenção de elevados níveis de atividade física, especialmente na infância. Contudo, os aspectos motivacionais que influenciam diretamente à prática de atividades físicas e esportivas, devem ser considerados a partir de seus diferentes tipos e domínios, os quais envolvem valorização a diversos aspectos como: status social, competência, apoio, amizades, competição, saúde, estética e recreação e lazer, variando de acordo com o instrumento utilizado ${ }^{8}$. Assim destaca-se não somente a relevância científica desse tipo de investigação, produzindo conhecimento com intuito de preencher uma lacuna existente na literatura, mas principalmente a importância social de pesquisas sobre motivação para a prática esportiva e de atividade física, onde o conhecimento científico produzido contribuirá para o conhecimento dos aspectos mais importantes para adoção de práticas de atividade física e esportes em cada população, possibilitando a formulação intervenções mais eficientes que contribuam para a alteração do quadro de inatividade física na atualidade ${ }^{8-10}$. A importância das pesquisas referentes à motivação para a prática de atividade física e esportiva reflete, deste modo, no crescimento substancial da produção científica mundial nas últimas décadas, inclusive com aumento no número de periódicos científicos indexados nas principais bases de dados internacionais que tratam dessa temática ${ }^{11,12}$. Assim, o presente estudo tem como objetivo revisar sistematicamente a produção científica sobre motivação para prática de atividades físicas e esportivas de crianças, contribuindo com a produção de conhecimento específico desta temática, com a população em questão, junto às bases de dados e metodologia selecionadas.

\section{Métodos}

Esta revisão foi realizada segundo as recomendações da Colaboração Cochrane ${ }^{13}$ e Preferred Reporting Items for Systematic Review and Meta-analyses: The PRISMA Statement ${ }^{14}$ e desenvolvida de maneira metódica, explícita e passível de reprodução, com uma pergunta clara, estratégia de busca e critérios de inclusão e exclusão bem definidos, visando nortear futuras investigações, sistematizando o conhecimento produzido $^{15,16}$.

Foram incluídos artigos científicos originais de base populacional ou escolar sem restrição de local da realização do estudo e de idioma; com amostra de crianças que não possuíam deficiência; com temática sobre motivação para a prática de atividade física e esportiva de crianças; com resumos e textos completos disponíveis na íntegra pelo meio on-line; publicados nos últimos 10 anos.

As bases de dados eletrônicas foram selecionadas de acordo com a área de conhecimento, área da saúde, além da relevância científica mundial dessas. Assim, foram utilizadas: MEDLINE (Medical Literature Analysis and Retrieval System on-line) via Pubmed, Scientific Eletronic Library Online (Scielo), Web of Science e SCOPUS (Elsevier) e a estratégia de busca incluiu os descritores propostos no Medical Subject Headings (MeSH) e seus correlatos: "Motivation", "Disincentives", "Disincentive", "Incentives", "Incentive”, "Sports", "Sport", "Athletics", "Athletic", "Motor Activity”, "Child”, "Children”. Foram incluídos estudos sobre motivação para a prática de atividade física e esportiva de crianças provenientes de qualquer região do mundo. Todas as estratégias de busca foram desenvolvidas no mês de janeiro de 2016.

A seleção dos estudos foi realizada por dois revisores, de forma independente, 
e iniciou por meio da análise dos títulos dos artigos identificados por meio da estratégia de busca, seguido pela análise dos resumos e, por fim, a análise do texto na íntegra dos artigos selecionados nas etapas anteriores. As discordâncias entre os revisores foram resolvidas por consenso.

Diante dessa sistemática, os dados extraídos foram: identificação da publicação, tipo de delineamento, faixa etária, tamanho da amostra, local da coleta dos dados, metodologia utilizada, instrumento utilizado para mensuração da motivação e seus resultados mais importantes. Após organização dos dados extraídos, as informações foram apresentadas de forma descritiva, por meio de tabelas.

\section{Resultados}

A busca permitiu identificar 838 artigos, sendo que após a avaliação, foram excluídos 377 estudos pela análise dos títulos e 278 pela análise dos resumos, em ambos os casos por não contemplarem a temática abordada e os objetivos deste estudo. Após a leitura na íntegra dos artigos selecionados, foram excluídos 165 estudos pelos seguintes motivos: não apresentavam as informações quanto à motivação para a prática de atividade física e/ou esportes; já haviam sido identificados em outras bases de dados; o tipo de pesquisa não era condizente; e a amostra não era composta por crianças. Deste modo, 18 estudos atenderam todos os critérios de inclusão e foram incluídos na revisão (Figura 1).

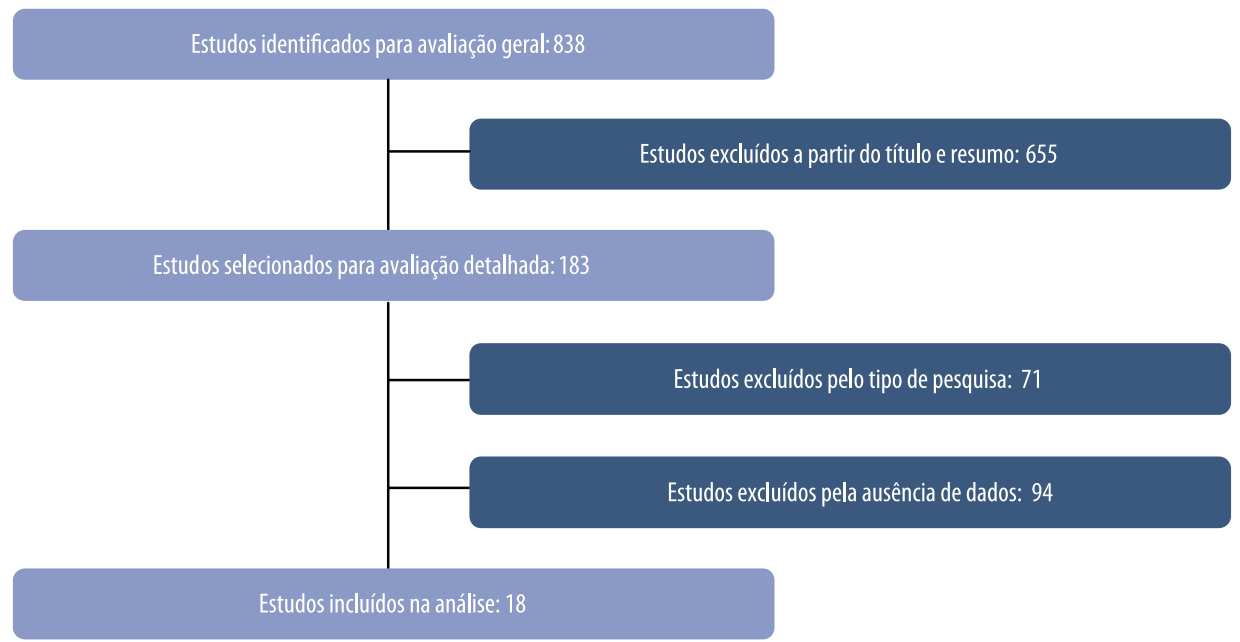

FIGURA 1 - Fluxograma dos estudos incluídos na revisão sistemática sobre motivação para a prática de atividades físicas e esportivas de crianças.

Além de apresentarem amostras compostas por crianças, 14 estudos também incluíam adolescentes como sujeitos de pesquisa ${ }^{9,10,17-28}$. As idades possuíram variação de $4^{23}$ a 25 anos $^{25}$.

Em relação a idade, dois estudos não apresentaram a variação da faixa etária, trazendo apenas as médias da idade de seus participantes ${ }^{17,29}$. Todos os estudos analisados foram realizados com ambos os sexos (Tabela 1). Os estudos foram realizados, em sua maioria, nos Estados Unidos da América $(n=6)^{9,17,19,25,29}$, seguido de Inglaterra $(\mathrm{n}=3)^{26,30,31}$, e Austrália $(\mathrm{n}=2)^{18,27}$ (Tabela 1).

No que diz respeito às características metodológicas, a maior parte dos estudos investigados utilizou delineamento transversal $(n=17)^{9,10,17-28,30-32}$, sendo que apenas um consistiu em um estudo longitudinal ${ }^{29}$. Quanto à análise dos dados, apenas três estudos deram enfoque qualitativo ${ }^{24,30,31}$, por meio de análise de conteúdo, enquanto que todos os demais com enfoque quantitativo, por meio de questioná- 
rios estruturados, com análise de dados por meio de estatística (Tabela 2).

TABELA 1 - Principais características sociodemográficas dos estudos sobre motivação para a prática de atividades físicas e esportivas em crianças.

\begin{tabular}{|c|c|c|c|}
\hline Autor/ano & País & Sexo & Faixa etária \\
\hline Salguero et al. (2003) & Espanha & Masculino e feminino & 8 a 22 anos \\
\hline Wilson et al. (2005) & EUA & Masculino e feminino & 10 a 13 anos \\
\hline Standage et al. (2005) & Inglaterra & Masculino e feminino & 11 a 14 anos \\
\hline Sirard et al. (2006) & EUA & Masculino e feminino & 7 a 25 anos \\
\hline Busey et al. (2007) & EUA & Masculino e feminino & 10 a 18 anos e seus pais \\
\hline Cox et al. (2008) & EUA & Masculino e feminino & 11,3 anos em média \\
\hline Keegan et al. (2009) & Inglaterra & Masculino e feminino & 7 a 11 anos \\
\hline Jago et al. (2009) & Inglaterra & Masculino e feminino & 10 e 11 anos \\
\hline Dollman et al. (2010) & Austrália & Masculino e feminino & 10 a 15 anos \\
\hline Simpkins et al.(2010) & EUA & Masculino e feminino & 8,7 anos em média \\
\hline Gao, et al. (2011) & EUA & Masculino e feminino & 10 a 14 anos \\
\hline Jõesaar et al. (2011) & Estônia & Masculino e feminino & 9 a 17 anos \\
\hline Stanley et al. (2012) & Austrália & Masculino e feminino & 10 a 13 anos \\
\hline Lämmle et al. (2013) & Alemanha & Masculino e feminino & 4 a 17 anos \\
\hline Seabra et al. (2013) & Portugal & Masculino e feminino & 8 a 10 anos \\
\hline Guedes e Neto (2013) & Brasil & Masculino e feminino & 12 a 18 anos \\
\hline Peykari et al. (2015) & Irã & Masculino e feminino & 10 a 19 anos \\
\hline Kopcakova et al. (2015) & $\begin{array}{c}\text { República Checa e } \\
\text { Eslováquia }\end{array}$ & Masculino e feminino & 11,13 e 15 anos \\
\hline
\end{tabular}

O tamanho das amostras nos estudos selecionados variou de $40^{31}$ a $9018^{22}$ sujeitos. Considerando que a pesquisa abrangeu estudos publicados nos últimos dez anos, identificou-se que 9, dos 15 artigos, foram publicados nos últimos cinco anos, o que indica que, dentre os estudos selecionados, houve um aumento de artigos nessa área nos últimos anos 18,19,21,23,27,29-32 (Tabela 2).

No que se refere aos métodos/técnicas para mensuração da motivação, pode-se verificar que os estudos utilizaram a aplicação de questionários $(\mathrm{n}=14)^{9,10,17-23,25-}$ $27,29,32$ e entrevistas $(n=4)^{24,28,30,31}$. Além disso, um dos estudos que aplicou questionário às crianças, também aplicou aos seus pais ${ }^{9}$ (Tabela 2).

Considerando que nesta revisão foram identificados 14 instrumentos para mensuração da motivação, deve-se salientar que sete estudos não apresentaram o instrumento utilizado, ressentindo-se de informações sobre autoria, validação, escopo, entre outros $(n=7)^{9,23,24,27-31}$.

Quanto ao sexo, os estudos demonstraram que as meninas valorizavam mais amizades, diversão e manutenção do físico, saúde e peso, enquanto os meninos valorizavam mais o desempenho e habilidades, competição, status social e amizades, ao passo que em relação à idade, as crianças mais novas valorizavam mais amizade e diversão e as crianças mais velhas concorrência, habilidades e competição.

A maior parte dos estudos demonstrou que as crianças incluídas em suas amostras valorizavam, em sua maioria, amizade, manutenção do físico, saúde e peso e status social. Ainda, apontaram como barreiras e dificultores principais à prática de atividades físicas e esportivas não escolher a modalidade o tipo de atividade, insegurança, instalações inadequadas, nível socioeconômico e falta de interesse de maneira geral. 
TABELA 2 - Principais características metodológicas dos estudos sobre motivação para a prática de atividades físicas e esportivas em crianças.

\begin{tabular}{|c|c|c|c|}
\hline Autor/ano & Delineamento & $\begin{array}{l}\text { Amostra } \\
(n)\end{array}$ & Instrumentos \\
\hline Salguero et al. (2003) & Transversal & 428 & $\begin{array}{l}\text { Inventário de motivação para participação (Gould, } \\
\text { Feltz e Weiss, 1985) }\end{array}$ \\
\hline Wilson et al. (2005) & Transversal & 51 & Entrevista com questionário não apresentado \\
\hline Standage et al. (2005) & Transversal & 950 & $\begin{array}{l}\text { Perceived locus of causality (Goudas, Biddle e Fox, } \\
\text { 1994), The amotivation subscale (Goudas et al., 1994) }\end{array}$ \\
\hline Sirard et al. (2006) & Transversal & 1692 & $\begin{array}{l}\text { Versão modificada do Participation Motivation } \\
\text { Questionnaire (Ewin g e Seefeldt, 1990) }\end{array}$ \\
\hline Busey et al. (2007) & Transversal & $\begin{array}{l}100 \text { pais } \\
138 \text { crianças }\end{array}$ & Questionário não apresentado \\
\hline Cox et al. (2008) & Transversal & 508 & $\begin{array}{c}\text { Duas subescalas de percepção do Perception of } \\
\text { Motivational Climate Scale (Biddle et al., 1995). Uma } \\
\text { sub-escala, Promotion of Learning by Teacher e The } \\
\text { Academic Self-Regulation Questionnaire (Ryan e } \\
\text { Connell, 1989) com modificações }\end{array}$ \\
\hline Keegan et al. (2009) & $\begin{array}{l}\text { Qualitativo - } \\
\text { transversal }\end{array}$ & 40 & $\begin{array}{c}\text { Entrevista - Main dimensions of motivated behaviour } \\
\text { reported (Roberts, 2001) }\end{array}$ \\
\hline Jago et al. (2009) & $\begin{array}{l}\text { Qualitativa - } \\
\text { transversal }\end{array}$ & 113 & $\begin{array}{c}\text { Entrevistas gravadas e transcritas com questões não } \\
\text { apresentadas }\end{array}$ \\
\hline Dollman et al. (2010) & Transversal & 1737 & $\begin{array}{l}\text { The Children's Physical Activity Correlates Scale } \\
\text { (Schaben et al. 2006) }\end{array}$ \\
\hline Simpkins et al. (2010) & Longitudinal & 987 & Questionário não apresentado \\
\hline Gao, et al. (2011) & Transversal & 194 & 25-item scale (Xiang e Lee, 2002) \\
\hline Jõesaar et al. (2011) & Transversal & 659 & $\begin{array}{l}\text { The Sport Motivation Scale (Pelletier, Fortier, } \\
\text { Vallerand, Tuson, Brière, e Blais, 1995) }\end{array}$ \\
\hline Stanley et al. (2012) & Transversal & 54 & Questionário não apresentado \\
\hline Lämmle et al. (2013) & Transversal & 4529 & Questionário não apresentado \\
\hline Seabra et al. (2013) & Transversal & 683 & $\begin{array}{c}\text { Versão curta do Children's Attraction to Physical } \\
\text { Activity Scale (Brustad, 1993) }\end{array}$ \\
\hline Guedes e Neto (2013) & Transversal & 1517 & $\begin{array}{l}\text { Versão em português do Participation Motivation } \\
\text { Questionnaire (Guedes e Silvério Netto, 2013) }\end{array}$ \\
\hline Peykari et al. (2015) & $\begin{array}{l}\text { Qualitativa - } \\
\text { Transversal }\end{array}$ & 98 & Entrevistas com questões não apresentadas \\
\hline $\begin{array}{l}\text { Kopcakova et al. } \\
\text { (2015) }\end{array}$ & Transversal & 9018 & $\begin{array}{l}\text { Health Behavior in School-aged Children (Currie et } \\
\qquad \begin{array}{l}\text { al., 2008) }\end{array}\end{array}$ \\
\hline
\end{tabular}

O apoio dos pais, treinadores e pares foi relatado em cinco estudos como um fator importante para motivação das crianças e sua prática de atividade físi$\mathrm{ca}^{18,21,26,27,32}$, sendo que o apoio dos pares foi considerado o mais importante em um desses mesmos estudos ${ }^{32}$. De maneira geral, os meninos receberam maiores influências positivas e apoio sobre a participação do que as meninas.

A motivação esteve relacionada ao nível de atividade física, nos estudos de Lämmle et al. ${ }^{23}$ e Simpkins et al. ${ }^{29}$, demonstrando que as crianças que possuíam maior motivação nos domínios estudados possuíam maior nível de atividade física, os demais não apresentaram esse tipo de comparativo.

O tipo de esporte foi considerado como importante motivador para a prática esportiva e de atividade física em três estudos ${ }^{10,17,28}$.

Os principais resultados de cada estudo incluído na presente revisão sobre motivação para a prática de atividades físicas e esportivas em crianças estão apresentados na Tabela 3 . 
TABELA 3 - Principais resultados identificados nos estudos incluídos quanto à motivação para a prática de atividades físicas e esportivas em crianças.

\begin{tabular}{|c|c|}
\hline Autor/ano & Principais resultados \\
\hline $\begin{array}{l}\text { Salguero et al. } \\
(2003)\end{array}$ & $\begin{array}{l}\text { Encontradas diferenças na motivação das crianças quanto à idade e o sexo. Meninas } \\
\text { valorizavam mais amizade e diversão e menos o status, assim como as crianças mais jovens (8- } \\
10 \text { anos), que ainda incluíram competir e desenvolver habilidades. Os resultados demonstram } \\
\text { que o tipo de esporte é extremamente relevante para os diferentes níveis de motivação. }\end{array}$ \\
\hline $\begin{array}{l}\text { Wilson et al. } \\
(2004)\end{array}$ & $\begin{array}{l}\text { Os meninos se sentiram mais motivados nas atividades em que tinham melhores desempenhos } \\
\text { e por demonstrar suas habilidades e como as meninas, valorizaram atividades com os amigos } \\
\text { e preferiram às atividades recreativas e de lazer e levantaram questões como: "Eu acho que } \\
\text { seria melhor escolher o que fazer". Evitar o tédio foi levantado, especialmente pelas meninas, } \\
\text { destancando ainda ocupar o tempo ocioso dos seus praticantes e diminuir o risco social. Manter } \\
\text { a forma física também pareceu um importante fator motivador para as meninas. }\end{array}$ \\
\hline $\begin{array}{l}\text { Standage et al. } \\
(2005)\end{array}$ & $\begin{array}{l}\text { Não houve grandes diferenças entre sexos. Aqueles que tiveram necessidade de apoio } \\
\text { apresentaram efeitos positivos sobre a motivação intrínseca }(\beta=0,68) \text { e regulação introjetada } \\
(\beta=0,36) \text {, e efeitos indiretos negativos sobre a regulação externa }(\beta=-0,35) \text { e desmotivação } \\
(\beta=-0,50) \text {. Necessidade de satisfação foi indiretamente previsto por percepções de autonomia } \\
(\beta=0,64) \text {, apoio de competência }(\beta=0,65) \text {, e apoio de parentesco }(\beta=0,69) \text {. Necessidade } \\
\text { de apoio teve efeitos indiretos positivos na satisfação e necessidade de regulamentos } \\
\text { motivacionais sobre a concentração }(\beta=0,52) \text {, afeto positivo }(\beta=0,62) \text {, e preferência por } \\
\text { tarefas desafiadoras }(\beta=0,28) \text {, enquanto um efeito indireto negativo foi observado entre } \\
\text { a necessidade de apoio e sentimentos de infelicidade }(\beta=-0,34) \text {. Efeitos indiretos positivos } \\
\text { surgiram por meio dos regulamentos de motivação para a satisfação de necessidades } \\
\text { de concentração }(\beta=0,72) \text {, afeto positivo }(\beta=0,85) \text {, e preferência por tarefas desafiadoras } \\
\text { ( } \beta=0,39) \text {, enquanto foi observado um efeito indireto negativo entre a necessidade satisfação e } \\
\text { sentimentos de infelicidade }(\beta=-0,46) \text {. }\end{array}$ \\
\hline $\begin{array}{l}\text { Sirard et al. } \\
(2006)\end{array}$ & $\begin{array}{l}\text { Para os meninos, os fatores de participação foram em ordem: competição, benefícios sociais } \\
\text { e fitness. Para as meninas foram: benefícios e habilidades sociais, competição e fitness. Para } \\
\text { ambos os sexos, as barreiras foram: falta de interesse, problemas de treinamento e tempo. } \\
\text { Participantes recentes de atividade física relataram mais tempo em atividades vigorosas } \\
\text { ( } p<0,01) \text {, alongamento }(p=0,03) \text {, e fortalecimento das atividades ( } p<0,01) \text { e menos tempo } \\
\text { assistindo televisão ( } p<0,01) \text {. A pontuação para o fator de fitness foi significativamente maior } \\
\text { para as meninas do que os meninos }(4,0 \pm 1,1 \text { vs } 3,8 \pm 1,0 \text {, respectivamente, } p<0,01) \text { e para } \\
\text { os participantes recentes do que os ex-participantes }(4,0 \pm 1,0 \text { vs } 3,2 \pm 1,7 \text {, respectivamente, } \\
p<0,01) \text {. }\end{array}$ \\
\hline $\begin{array}{l}\text { Busey et al. } \\
(2007)\end{array}$ & $\begin{array}{l}\text { "Desenvolvimento de hábitos saudáveis" e "tornar-se fisicamente apto e saudável" foram } \\
\text { os fatores motivadores melhor considerados para pais e filhos. "Ajudar o meu filho a perder } \\
\text { ou ganhar peso", foi o segundo item menos motivante para ambos. "Tornar-se um atleta } \\
\text { profissional", foi o item menos motivante para os pais e "se divertir e fazer amigos" foi o menos } \\
\text { motivante para as crianças. }\end{array}$ \\
\hline $\begin{array}{l}\text { Cox et al. } \\
(2008)\end{array}$ & $\begin{array}{l}\text { Os alunos apoiados pelos professores se sentiram competentes a participar das atividades } \\
\text { físicas, eram mais socialmente relacionados na aula e foram mais autodeterminados e } \\
\text { motivados. Desmotivados por não escolher as atividades das aulas. As relações de domínio, } \\
\text { percepção de competência e parentesco foram negativas }(p<0,01) \text {. O domínio demonstrava } \\
\text { relações significativas e positivas com as percepções de competência, autonomia e } \\
\text { relacionamento. A relação direta entre o domínio e motivação autodeterminada foi } \\
\text { significativa. Nas aulas de educação física o contexto e as relações sociais demonstraram- } \\
\text { se maiores aspectos motivacionais do que os de competência e autonomia. O clima } \\
\text { motivacional das aulas e as atitudes do professor foram considerados grandes preditores para } \\
\text { a motivação autodeterminada. }\end{array}$ \\
\hline $\begin{array}{l}\text { Keegan et al. } \\
\text { (2009) }\end{array}$ & $\begin{array}{l}\text { Os pais e treinadores exerceram influências por meio de seus estilos de liderança, respostas } \\
\text { afetivas e comportamentos pré-desempenho na motivação dos participantes. Os pares } \\
\text { influenciaram principalmente na motivação dos participantes por meio de comportamentos } \\
\text { competitivos, comportamentos de colaboração, comunicações de avaliação e por meio de } \\
\text { suas relações sociais. }\end{array}$ \\
\hline $\begin{array}{l}\text { Jago et al. } \\
\text { (2009) }\end{array}$ & $\begin{array}{l}\text { A atividade física foi percebida como um atributo positivo e ligado ao status social entre } \\
\text { os meninos especialmente para aceitação dos pares. Entre as meninas a associação entre } \\
\text { atividade física e capacidade de status social era mais complexa. Algumas participantes } \\
\text { relataram que a baixa capacidade para atividade poderia ser percebida como desejável em } \\
\text { alguns grupos sociais. Os participantes relataram que os amigos fornecem suporte para iniciar } \\
\text { a atividade física por meio de coparticipação, modelo e suporte verbal. Prazer foi o fator mais } \\
\text { importante na manutenção da participação em atividade com a participação em atividade } \\
\text { com os amigos. }\end{array}$ \\
\hline
\end{tabular}


.. continua

\begin{tabular}{|c|c|}
\hline Autor/ano & Principais resultados \\
\hline $\begin{array}{l}\text { Dollman et al. } \\
\text { (2010) }\end{array}$ & $\begin{array}{l}\text { A participação esportiva foi maior entre as crianças de altas classes econômicas em } \\
\text { comparação aos de baixa. Todos os indicadores, exceto "eu sou capaz?" foram associados } \\
\text { positivamente com a participação no esporte entre ambos os sexos. As crianças com altos } \\
\text { níveis socioeconômicos registraram menor número de barreiras. As meninas de classes sociais } \\
\text { mais baixas relataram menos apoio dos pais e condições instrumentais para praticar atividades. } \\
\text { Não foram observadas diferenças entre meninos e meninas na proporção de pelo menos um } \\
\text { esporte organizado nos últimos } 12 \text { meses. Por outro lado, os meninos relataram influências } \\
\text { mais positivas sobre a participação do esporte do que as meninas em todas as questões. }\end{array}$ \\
\hline $\begin{array}{l}\text { Simpkins et al. } \\
(2010)\end{array}$ & $\begin{array}{l}\text { As crianças que não participaram de qualquer atividade durante a escola primária foram } \\
\text { mais passíveis de baixa motivação e baixa participação em ambos os domínios durante } \\
\text { a adolescência. As crianças que foram focadas em esportes na escola primária eram mais } \\
\text { suscetíveis a baixa motivação na adolescência. As que praticavam atividades físicas na escola } \\
\text { tinham crenças motivacionais mais elevadas do que aquelas que não praticavam, ainda, aquelas } \\
\text { que participaram durante os três anos do ensino elementar, tiveram crenças motivacionais } \\
\text { posteriores mais altas do que aquelas que participaram durante um ou dois anos. }\end{array}$ \\
\hline $\begin{array}{l}\text { Gao, et al. } \\
(2011)\end{array}$ & $\begin{array}{l}\text { A autoeficácia foi significativamente e positivamente relacionada com as quatro metas de } \\
\text { realização e percepções de climas de motivação }(r=0,18-0,58, p<0,05) \text {. Além disso, a atividade } \\
\text { física dos estudantes foi associada à autoeficácia e percepções de climas motivacionais. As } \\
\text { influências parecem ser um grande motivador especialmente dos professores e treinadores. }\end{array}$ \\
\hline $\begin{array}{l}\text { Jõesaar et al. } \\
\text { (2011) }\end{array}$ & $\begin{array}{l}\text { Os desistentes desmotivados apresentaram menor competência, relacionamento, autonomia } \\
\text { e necessidade de satisfação, e perceberam menos apoio autonomia dos pais e eram } \\
\text { menos intrinsecamente motivados. Atletas com até um ano de treinamento relataram } \\
\text { significativamente menor esforço e conflito com colegas, relacionamento e necessidade } \\
\text { de satisfação e motivaçãa externa do que atletas com } 1 \text { a } 3 \text { anos e }>3 \text { anos em formação. } \\
\text { Os persistentes relataram uma maior sensação de satisfação percebida, necessidade de } \\
\text { autonomia, competência e status social do que os participantes que não continuaram. A } \\
\text { motivaçãa extrínseca e o apoio com autonomia por parte dos treinadores não diferiu entre os } \\
\text { grupos persistentes e que abandonaram a prática. }\end{array}$ \\
\hline $\begin{array}{l}\text { Stanley et al. } \\
(2012)\end{array}$ & $\begin{array}{l}\text { Oportunidades para a prática são facilitadores e o clima foi um fator de motivação. Valorização } \\
\text { do: "porque é divertido" ou "eu gosto". Os aspectos sociais também foram bem relacionados, } \\
\text { bem como as atitudes comportamentais e sentimentos pelo jogo. Na hora do almoço a } \\
\text { valorização foi a melhoria das competências ou a obtenção de prática. "Algo para fazer" } \\
\text { também foi bem relacionado. A liberdade das crianças para "fazerem suas próprias regras" foi } \\
\text { motivante. A categoria "influência dos pares" foi um dos fatores mais importantes que facilitam } \\
\text { o jogo, assim como "aceitação dos colegas". O apoio de professores não foi considerado tão } \\
\text { importante quanto à influência dos pares. }\end{array}$ \\
\hline $\begin{array}{l}\text { Lämmle et al. } \\
\text { (2013) }\end{array}$ & $\begin{array}{l}\text { A motivação intrínseca foi associada com mais atividade física, maior qualidade de vida } \\
\text { e menos problemas psicopatológicos. O nível de atividade física dos pais e colegas foi } \\
\text { positivamente associado à motivação intrínseca de ser fisicamente ativo e nível da criança. }\end{array}$ \\
\hline $\begin{array}{l}\text { Seabra et al. } \\
\text { (2013) }\end{array}$ & $\begin{array}{l}\text { Prazer de participação em atividades físicas vigorosas foi associado positivamente com o nível } \\
\text { de atividade física. Percepção de aceitação pelos colegas nas atividades e incentivo dos pais } \\
\text { relacionou-se às meninas e competência física aos meninos. }\end{array}$ \\
\hline $\begin{array}{l}\text { Guedes e Neto } \\
(2013)\end{array}$ & $\begin{array}{l}\text { Valorização de desenvolvimento de habilidades }(4,2 \pm 0,8) \text { e manter a forma }(4,0 \pm 1,0) \text {. } \\
\text { Os motivos com as mais baixas classificações foram diversão }(2,8 \pm 1,2) \text { e status }(2,7 \pm 1,1) \text {. } \\
\text { Em seguida foram amizade }(3,9 \pm 1,1) \text {, trabalho em equipe }(3,8 \pm 1,0) \text {, competição }(3,4 \pm \\
\text { 1,0) e emoção }(3,2 \pm 1,1) \text {. Meninos valorizaram mais concorrência e desenvolvimento de } \\
\text { competências, enquanto as meninas tiveram trabalho em equipe e amizade. Com relação } \\
\text { à idade, status e diversão foram mais valorizadas pelos mais novos e concorrência e o } \\
\text { desenvolvimento de competências para os mais velhos. }\end{array}$ \\
\hline $\begin{array}{l}\text { Peykari et al. } \\
(2015)\end{array}$ & $\begin{array}{l}\text { Valorização da manutenção da saúde, apesar de não ser prioridade, com foco na manutenção } \\
\text { do peso. Destaque para as barreiras: segurança e instalações, preguiça, falta de atitude efetiva, } \\
\text { autoconfiança, e hábitos. }\end{array}$ \\
\hline $\begin{array}{l}\text { Kopcakova et } \\
\text { al. (2015) }\end{array}$ & $\begin{array}{l}\text { Meninas valorizaram mais motivos sociais e de saúde, enquanto os meninos valorizaram mais } \\
\text { a realização pessoal e conquistar bons resultados. }\end{array}$ \\
\hline
\end{tabular}

\section{Discussão}

A literatura demonstra-se bastante vasta quanto à motivação de crianças. Contudo, a maior parte dos estudos investiga a motivação frente a uma determinada questão, tais como: oportunidades para a prática, tempo e tipo de prática, sendo poucos os estudos encontrados nas bases de dados selecionadas que abrangessem especificamente a motivação para a prática esportiva e de atividade física em seu 
contexto geral. Deste modo, os estudos selecionados analisam a motivação para a prática esportiva e de atividade física de forma geral, conjuntamente a outras variáveis, sendo essas: sexo mais motivado, faixa etária com mais motivação para a prática, local de moradia mais propício à motivar a prática de atividades físicas e esportivas, condição socioeconômica em que está inserido o sujeito mais motivado, tipo de motivação dos sujeitos, qualidade de vida frente aos aspectos motivacionais, motivação intrínseca e extrínseca, desmotivação ou amotivação, hábitos e condições de saúde frente à motivação para a prática de atividades físicas e esportivas.

Alguns aspectos que envolvem a motivação constituem padrões que são decorrentes das necessidades psicológicas e do ambiente o qual o sujeito está inserido, como por exemplo, a desmotivação, que consiste na ausência de intenção, desvalorização da atividade e percepção de falta de controle pessoal; a motivação intrínseca, como interesse, satisfação e prazer, inerentes à atividade; e a motivação extrínseca que seria a realização da atividade como uma obrigação ou como um meio para obter algo externo ${ }^{33,34}$. Nos estudos incluídos nesta revisão a motivação intrínseca para prática de atividade física foi fortemente ligada às questões referentes à qualidade de vida e saúde, demonstrando-se um importante fator para a realização de atividades ${ }^{23}$. Na motivação extrínseca, especialmente durante a infância, as influências parecem ser um grande motivador, ressaltando-se principalmente o apoio dos professores, treinadores, pais e pares ${ }^{19,30,31}$.

De maneira geral, os estudos incluídos nesta revisão corroboraram com a literatura especializada, demonstrando que os aspectos motivacionais mais relevantes foram referentes às interações sociais e amizades e manutenção física por estética e saúde, destacando que os fatores sociais e de relacionamento são significativamente motivantes para crianças de todo o mundo ${ }^{17,22,30-32,35}$.

Para as meninas a valorização maior também incluiu a diversão e lazer e menos tanto o status social, enquanto os meninos também valorizaram o desempenho e habilidades, competição e o status social. Esses achados são justificados por Sirard, Pfeiffer e Pate ${ }^{25}$ que concluíram que os meninos se sentem mais motivados a participar de atividades competitivas, enquanto as meninas se sentem mais atraídas por atividades de participação, com cunho de benefício social.

Em relação à idade, as crianças mais novas valorizavam mais amizade e diversão e as crianças mais velhas concorrência, habilidades e competição, o que é comprovado pelo fato da idade ser considerada inversamente proporcional ao nível de atividade física, ou seja, o nível de atividade física tende a diminuir com o aumento da idade $e^{3,4,36}$. Assim, justifica-se o fato das crianças iniciarem seus hábitos de atividade física por diversos fatores, especialmente sociais e de lazer e permanecerem com o hábito da prática de atividade física quando mais velhos por estarem envolvidos com treinamento e competições, de forma sistematizada e organizada, destacando que a literatura demonstra que estes tipos de atividade são mais eficazes para a manutenção dos níveis de atividade física ${ }^{37,38,39}$. Ressaltando que os sujeitos que praticavam atividade há menos tempo apresentavamse mais motivados a participar e desenvolver habilidades ${ }^{25}$.

Nos estudos apresentados, a motivação esteve relacionada ao nível de atividade física demonstrando que as crianças mais motivadas possuíam maior nível de atividade física ${ }^{23,29}$. No entanto, não é possível generalizar quanto à atividade a ser realizada já que o tipo de atividade foi considerado um importante fator para participação e continuidade de participação $\mathrm{O}^{10,17,28}$.

As condições e oportunidades para a prática de atividade física e esportiva são 
essenciais para a participação e também foram apresentados como aspectos motivacionais importantes. O nível socioeconômico mais alto foi um facilitador para a motivação e prática de atividade física, ofertando maiores oportunidades para as práticas e que essas oportunidades são essenciais para atingir maiores níveis de atividade física ${ }^{40-42}$. Além disso, estudos ainda demonstram que o local e os equipamentos para a prática de atividade física e esportiva são aspectos motivacionais que influenciam diretamente a prática da atividade, podendo muitas vezes se tornar uma barreira para essa ${ }^{18,24,27}$.

O incentivo e apoio dos familiares, pares e treinadores foi considerado como um importante fator para a motivação e participação em atividades físicas e esportivas $18,21,26,27,32$, sendo ainda mais prevalentes para as meninas, que apesar de sentirem mais necessidade de apoio, recebem menos apoio, especialmente da família ${ }^{24,32}$.

Diante do exposto, pode-se apontar a necessidade de estudos desenvolvidos com crianças brasileiras que tragam informações complementares em relação à motivação para a prática de atividade física e esportiva. Essa afirmação sustenta-se nos achados desta revisão, demonstrando que apenas um dos estudos selecionados foi realizado no Brasil, apesar da produção científica da área vir aumentando nos últimos anos, especialmente aqueles com delineamentos transversais, bem como com utilização de diferentes questionários e entrevistas. E que, além disso, sejam realizados mais estudos longitudinais sobre prática esportiva e aspectos motivacionais, para verificar a influência da motivação para a prática de atividades físicas e esportivas frente à adoção de hábitos de saúde e de atividade física à longo prazo, tanto no que diz respeito às questões de qualidade de vida quanto ao potencial de transformação social do esporte, ocupando o tempo ocioso dos seus praticantes e diminuindo o risco social, como identificado no estudo de Wilson et al. ${ }^{28}$.

Considera-se de grande relevância científica o desenvolvimento de estudos a respeito da motivação para a prática esportiva, bem como revisões sistemáticas que visem abranger todos os aspectos motivacionais, complementando o presente estudo. A literatura especializada identificada por meio das bases de dados selecionadas confirma a importância da produção na área, demonstrando crescimento da produção científica. No entanto, também é válida a utilização do conhecimento adquirido em tais pesquisas para realização de intervenções junto ao público alvo, realizando atividades mais adequadas às necessidades e preferências da população, tornando-se, desta maneira, estratégias mais eficazes de obtenção dos benefícios da adoção de hábitos saudáveis.

Diante da importância da prática de atividade física pode-se considerar que os programas de atividade física e esportes são ótimas ferramentas para promoção de hábitos saudáveis, bem como extinção de comportamentos sedentários prejudiciais. Assim, a eficácia desse tipo de programa é ofertar um tipo de atividade que esteja de acordo com as preferências quanto ao tipo de atividade do público a que se destina, com possibilidade de convívio social, com orientações e foco às questões de saúde e manutenção da forma física, com possibilidade de competição e que de alguma forma, envolva a família e conte com o apoio dos pais, maximizaria a participação, permanência e consequentemente os resultados, contribuindo com a reversão do quadro de inatividade física estabelecido ${ }^{9,10,25}$.

\section{Contribuiç̧ão dos autores}

Cada autor contribuiu individual e significantemente para o desenvolvimento deste artigo. G. G. Bento: redação do artigo, concepção do trabalho, revisão crítica do 
seu conteúdo intelectual e análise dos dados; E. G. Ferreira: análise e interpretação dos dados, aprovação final da versão do manuscrito a ser publicado; F. G. da Silva: aprovação final da versão do manuscrito a ser publicado, redação do artigo, análise e interpretação dos dados; P. H. Mattana: redação e revisão do artigo, aprovação da versão a ser publicada; R. da Silva: contribuição substancial na concepção e delineamento do trabalho, interpretação dos dados, revisão crítica do seu conteúdo intelectual.

\section{Referências}

1. Hallal P, Andersen LB, Bull FC, Guthold R, Haskell W, Ekelund U. Global physical activity levels: surveillance progress, pitfalls, and prospects. Lancet. 2012;380:247-57.

2. Silva KS, Lopes AS, Silva FM. Atividade física no deslocamento à escola e no tempo livre em crianças e adolescentes da cidade de João Pessoa, PB, Brasil. Rev Bras Ciên e Mov. 2007;15(3):61-70.

3. Alves JGB. Atividade física em crianças: promovendo a saúde do adulto. Rev. Bras. Saúde Mater Infant. 2003;3(1):5-6.

4. Khoo S, Al-Shamli AK. Leisure-time physical activity and physical fitness of male adolescents in Oman. Asia Pac J Public Health. 2012;24(1):128-35.

5. Gonçalves H, Hallal PC, Amorim TC, Araújo CLP, Menezes AMB. Fatores socioculturais e nível de atividade física no início da adolescência. Rev Panam Salud Publ. 2007;22(4):246-53.

6. Rennie KL, Johnson L, Jebb SA. Behavioural determinants of obesity. Best Pract Res Clin Endocrinol Metab. 2005;19(3):343-58.

7. Ferreira VA, Magalhães R. Obesidade e pobreza: o aparente paradoxo. Um estudo com mulheres da Favela da Rocinha, Rio de Janeiro, Brasil. Cad de Saúde Púb. 2005;21(6):1792-800.

8. Silva HGV, Chiara VL, Barros ME, Rêgo AL, Ferreira A, Pitasi BAI, et al. Diagnosing the nutritional status of schoolchildren: a comparison between brazilian and international criteria. J Pediatr. 2008;84(6):550-5.

9. Busey SL, Batten CG, Young CC, Bragg DS. A survey of inner city youth and their parents about participation in sports. WMJ. 2007;106(6):307-11.

10. Salguero A, Gonzalez-Boto R, Tuero C, Márquez S. Development of a Spanish version of the Participation Motivation Inventory for young competitive swimmers. Percept Mot Skills. 2003;96:637-46.

11. Barreto ML. Crescimento e tendência da produção científica em epidemiologia no Brasil. Rev Saúde Públ. 2006;40:79-85.

12. Castro F. Questão de qualidade. Rev Bras Cir Cardiovasc. 2009;24(2):258-60.

13. Higgins JPT, Green S. Cochrane handbook for systematic reviews of interventions. Version 5.1.0. 2011. [acesso em 03 set. 2015]. Disponível em: http://handbook.cochrane.org

14. Moher D, Liberati A, Tetzlaff J, Altman DG. Preferred reporting items for systematic reviews and meta-analyses: The PRISMA Statement. Ann Intern Med. 2009;151(4):264-9.

15. Clarke M, Horton R. Bringing it all together: Lancet-Cochrane collaborate on systematic reviews. Lancet. 2001;357(9270):1728.

16. Sampaio RF, Mancini MC. Systematic review studies: a guide for careful synthesis of the scientific evidence. Rev Bras Fisioter. 2007;11(1):77-82.

17. Cox A, Duncheon N, McDavid L. Peers and teachers as sources of relatedness perceptions, motivation, and affective responses in physical education. Res Q Exerc Sport. 2009;80(4):765-73.

18. Dollman J, Lewis NR. The impact of socioeconomic position on sport participation among South Australian youth. J Sci Med Sport. 2010;13(3):318-22.

19. Gao Z, Lochbaum M, Podlog L. Self-efficacy as a mediator of children's achievement motivation and in-class physical activity. Percept Mot Skills. 2011;113(3):969-81.

20. Guedes DP, Netto JE. Sport participation motives of young brazilian athletes. Percept Mot Skills. 2013;117(3):742-59.

21. Jõesaar H, Hein V. Psychosocial determinants of young athletes continued participation over time. Percept Mot Skills. 2011;113(1):51-66.

22. Kopcakova J, Veselska ZD, Geckova AM, Kalman M, Dijk JPV, Reijneveld SA. Do motives to undertake physical activity relate to physical activity in adolescent boys and girls? Int J Environ Res Public Health. 2015;12(7):7656-66.

23. Lämmle L, Woll A, Mensink GB, Bös K. Distal and proximal factors of health behaviors and their associations with health in children and adolescents. Int J Environ Res Public Health. 2013;10(7):2944-78. 
24. Peykari N, Eftekhari MB, Tehrani FR, Afzali HM, Hejazi F, Atoofi MK, et al. Promoting physical activity participation among adolescents: the barriers and the suggestions. Int J Prev Med. 2015;6(1):6-12.

25. Sirard JR, Pfeiffer KA, Pate RR. Motivational factors associated with sports program participation in middle school students. J Adol Health. 2006;38(6):696-703.

26. Standage M, Duda JL, Ntoumanis N. A test of self-determination theory in school physical education. Br J Educ Psycholv. 2005;75(3):411-33.

27. Stanley RM, Boshoff K, Dollman J. Voices in the playground: a qualitative exploration of the barriers and facilitators of lunchtime play. J Sci Med Sport. 2012;15(1):44-51.

28. Wilson DK, Williams J, Evans A, Mixon G, Rheaume C. Brief report: A qualitative study of gender preferences and motivational factors for physical activity in underserved adolescents. J Pediat Psychol. 2005;30(3):293-7.

29. Simpkins SD, Vest AE, Becnel JN. Participating in sport and music activities in adolescence: the role of activity participation and motivational beliefs during elementary school. J Youth Adol. 2010;39:1368-86.

30. Jago R, Brockman R, Fox KR, Cartwright K, Page AS, Thompson JL. Friendship groups and physical activity: qualitative findings on how physical activity is initiated and maintained among 10-11 year old children. Int J Behav Nutr Phys Act. 2009;6(1):1-9.

31. Keegan RJ, Harwood CG, Spray CM, Lavallee DE. A qualitative investigation exploring the motivational climate in early career sports participants: Coach, parent and peer influences on sport motivation. Psychol Sport Exerc. 2009;10(3):361-72.

32. Seabra AC, Seabra AF, Mendonça DM, Brustad R, Maia JA, Fonseca AM, et al. Psychosocial correlates of physical activity in school children aged 8-10 years. Eur J Pub Health. 2013;23(5):794-8.

33. Guimarães SER, Bzuneck JA, Sanches SF. Psicologia educacional nos cursos de licenciatura: a motivação dos estudantes. Psicol Esc Educ. 2002;6(1):11-19.

34. Ryan RM, Connell JP, Deci EL. A motivational analysis of self-determination and self-regulation in education. In: Ames C.; Ames R. (Org.). Research on Motivation in Education. New York: Academic Press; 1985. p.16-31.

35. Mageau GA, Robert JV. The coach-athlete relationship: A motivational model. J Sports Sci. 2003;21(11):883-904.

36. Tenório MCM, Barros MVG, Tassitano RM, Bezerra J, Tenório JM, Hallal PC. Atividade física e comportamento sedentário em adolescentes estudantes do ensino médio. Rev Bras Epidemiol. 2010;13(1):105-17.

37. Francis SL, Letuchy EM, Levy SM, Janz KF. Sustained effects of physical activity on bone health: iowa bone development study. Bone. 2014;63:95-100.

38. Kwon S, Janz KF, Letuchy EM, Burns TL, Levy SM. Developmental trajectories of physical activity, sports, and television viewing during childhood to young adulthood: Iowa bone development study. JAMA Pediatr. 2015;169(7):666-72.

39. Wagnsson S, Lindwall M, Gustafsson H. Participation in organized sport and self-esteem across adolescence: the mediating role of perceived sport competence. J Sport Exerc Psycho. 2014;36(6):584-94.

40. Guedes DP, Guedes JERP, Barbosa DS, Oliveira JA. Níveis de prática de atividade física habitual em adolescentes. Rev Bras Med Esp. 2001;7(6):187-99.

41. Farias Júnior JC, Lopes AS. Comportamentos de risco relacionados à saúde em adolescentes. Rev Bras Cie Mov. 2004;12(1):7-12.

42. Hallal P, Bertoldi AD, Gonçalves H, Victora CG. Prevalência de sedentarismo e fatores associados em adolescentes de 10-12 anos de idade. Cad Saúde Públ. 2006;22(6):1277-87.

Endereço para

correspondência

Gisele Graziele Bento

giselegbento@gmail.com
Rua Pascoal Simone, 358

CEP: 88080-350

Florianópolis, SC, Brasil.
Recebido

Revisado

Aprovado
$20 / 04 / 2016$ 20/08/2016

$17 / 10 / 2016$ 